Review Article

\title{
A Comprehensive Biological and Clinical Perspective Can Drive a Patient-Tailored Approach to Multiple Myeloma: Bridging the Gaps between the Plasma Cell and the Neoplastic Niche
}

\author{
Antonio Giovanni Solimando, ${ }^{1}$ Angelo Vacca, ${ }^{1}$ and Domenico Ribatti ${ }^{2}{ }^{2}$ \\ ${ }^{1}$ Department of Biomedical Sciences and Human Oncology, \\ Section of Internal Medicine "Guido Baccelli" University of Bari Aldo Moro, Piazza Giulio Cesare, 11, 70124 Bari, Italy \\ ${ }^{2}$ Department of Basic Medical Sciences, Neurosciences and Sensory Organs, University of Bari Medical School, Bari, Italy \\ Correspondence should be addressed to Domenico Ribatti; domenico.ribatti@uniba.it
}

Received 21 December 2019; Revised 17 February 2020; Accepted 10 April 2020; Published 18 May 2020

Guest Editor: Klaus Podar

Copyright (c) 2020 Antonio Giovanni Solimando et al. This is an open access article distributed under the Creative Commons Attribution License, which permits unrestricted use, distribution, and reproduction in any medium, provided the original work is properly cited.

\begin{abstract}
There is a broad spectrum of diseases labeled as multiple myeloma (MM). This is due not only to the composite prognostic risk factors leading to different clinical outcomes and responses to treatments but also to the composite tumor microenvironment that is involved in a vicious cycle with the MM plasma cells. New therapeutic strategies have improved MM patients' chances of survival. Nevertheless, certain patients' subgroups have a particularly unfavorable prognosis. Biological stratification can be subdivided into patient, disease, or therapy-related factors. Alternatively, the biological signature of aggressive disease and dismal therapeutic response can promote a dynamic, comprehensive strategic approach, better tailoring the clinical management of highrisk profiles and refractoriness to therapy and taking into account the role played by the MM milieu. By means of an extensive literature search, we have reviewed the state-of-the-art pathophysiological insights obtained from translational investigations of the MM-bone marrow microenvironment. A good knowledge of the MM niche pathophysiological dissection is crucial to tailor personalized approaches in a bench-bedside fashion. The discussion in this review pinpoints two main aspects that appear fundamental in order to gain novel and definitive results from the biology of MM. A systematic knowledge of the plasma cell disorder, along with greater efforts to face the unmet needs present in MM evolution, promises to open a new therapeutic window looking out onto the plethora of scientific evidence about the myeloma and the bystander cells.
\end{abstract}

\section{Introduction}

Multiple myeloma (MM) is an incurable haematological malignancy characterized by a clonal proliferation of plasma cells that accumulate preferentially in the bone marrow (BM). It accounts for $1 \%$ of all cancers and $10 \%$ of all haematological malignancies. Resistance to chemotherapy poses one of the main challenges in MM management [1]. Indeed, although advances in MM pathophysiological deconvolution and therapeutic knowledge, $\mathrm{MM}$ is still an incurable disease [2]. According to Durie-Salmon (D\&S) clinical staging, MM patients can be stratified based on available clinical parameters, such as haemoglobin, serum calcium value, X-ray bone study, immunoglobulins, and urine light chains. These parameters may be useful to foresee the patient characteristics from a biological standpoint, in order to predict therapy response and estimate the MM load [3]. Nonetheless, the D\&S is affected by observer-related bias in quantifying lytic lesions, and since 2005, it has been replaced by the International Staging System (ISS), which is based only on the combination of two parameters, namely, $\beta 2$-microglobulin and albumin [4]. Nowadays, sensitivity and specificity of bone disease identification have improved, thanks to the widespread use of computed tomography and of functional imaging such as magnetic resonance and positron-emission tomography (PET scan) [1]. Moreover, among the three stages, data on ISS stage III, associated with the poorest outcome, are now available. Since cytogenetics, 
evaluated by fluorescent in situ hybridization (FISH), is also a major prognostic factor, a new paradigm shift in patient risk stratification has incorporated the three recurrent genetic abnormalities, the $t(4 ; 14)$, deletion $(17 \mathrm{p})$, and $t(14 ; 16)$, that are mostly associated with a poorer outcome. These are used, along with the clinical and laboratory parameters, in order to gain a more reliable MM risk classification according to the revised ISS (R-ISS) [5]. Undoubtedly, in MM, the genomic landscape has a strong impact on patient outcome and response to therapy [6-8]. Nevertheless, the disease aggressiveness is not only linked to multistep genetic events but also to the MM microenvironment and the MM bystander cells, involved within the tumor niche in a vicious cycle that leads to MM evolution into more complex pathological architecture [9]. BM microenvironment-mediated drug resistance is the main mechanism allowing MM to evade the effects of conventional and new drugs [10]. To date, a plethora of pathophysiological mechanisms has been dissected, but potential targets considered suitable for therapeutic interventions aimed at interfering with the mutual interactions between the clonal plasma cells and the tumor milieu have proven largely unsatisfactory [11]. In this scenario, we have carried out an extensive literature review to probe the novel insights available from translational investigations, in order to reach a deeper understanding of the emerging therapeutic window from a bench-to-bedside standpoint.

\section{The Role of the Bone Marrow Microenvironment: Novel Molecular Dependencies in Multiple Myeloma}

Signals from the bone marrow microenvironment play a pivotal role in supporting MM cell growth, spread, and survival, as well as MM progression [12]. In the bone marrow microenvironment, the cellular compartment consists of hematologic and nonhematopoietic cells such as stromal cells, fibroblasts, osteoblasts, osteoclasts, endothelial cells (ECs), B cells, T cells, natural killer (NK) cells, macrophages, mast cells, and myeloid-derived suppressor cells (MDSCs). During MM development, MM cells can affect the $\mathrm{BM}$ cells through cell-cell contact or the secretion of soluble factors to build up a favorable microenvironment. MM cells adhere to bone marrow stromal cells (BMSCs) and trigger many pathways in the latter, resulting in the transcription and secretion of multiple cytokines such as interleukin-6 (IL-6), insulin-like growth factor-1 (ILGF-1), vascular endothelial growth factor (VEGF), and stromal cell-derived factor- $1 \alpha$ (SDF-1 $\alpha)$ which mediate MM cell growth, proliferation, survival, and drug resistance [13]. Next, MM cells educate the bone marrow cells to support neoplastic cell growth, survival, and the acquisition of drug resistance resulting in disease relapse, conferring a survival advantage. The tumor microenvironment is recognized as one of the leading factors promoting chemoresistance, but the mechanisms responsible for this effect are still largely obscure [14].
Current studies are focused on the bone marrow microenvironment and inflammatory cells as attractive druggable targets [15]. The MM physiology offers a wide range of targeting opportunities, which can be useful in chemotherapeutics for devising more personalized therapy for MM patients [16]. For example, increased BM hypoxia is associated with increased recirculation of MM plasma cells (MM-PCs). Oxygen delivery decrease, by enhancing hypoxia-inducible factor-2 alpha (HIF-2 $\alpha$ ) activity, induces MM-PC chemokine ligand 12 (CXCL12) upregulation, with a diminished migration toward CXCL12 and reduced adhesion to mesenchymal stromal cells in vitro. HIF- $2 \alpha$ also strongly induced the expression of chemokine receptor 1 (CCR1) in MM-PCs. CCR1 enhances MM-PC dissemination toward CCL3, while decreasing the MM-PC motility reaction to CXCL12. Additionally, CCR1 upregulation by MM-PCs was correlated with a poor outcome in newly diagnosed MM subjects and associated with enhanced circulating MM-PCs in these individuals. Taken together, these data suggest a role for hypoxia-mediated CCR1 upregulation in driving the egress of MM-PCs from the BM. Targeting CCR1 may be a novel strategy to prevent dissemination and overt relapse in MM [17].

Mesenchymal stem cells (MSCs), one of the main cell components within the BM milieu, can disseminate toward primary tumors and metastatic sites, implying that these cells might modulate tumor growth and metastasis [13]. Myeloma-derived MSCs can deeply impact the disease homeostasis. Therefore, MSCs do not represent bystanders in the BM niche but rather dynamic actors in the MM biology. MSCs can represent a novel target to develop the next generation of therapy in cancer, both by in vitro engineering as antitumor carrier to the tumor sites. MM is no exception to this principle [18]. MSCs were lentivirally engineered with osteoprotegerin (OPG) in preclinical models aimed to halt MM-related skeletal lesions [19]. The first-in-class proteasome inhibitor bortezomib shapes the tumor-friendly MM environment by inducing bone matrix remodelling [20] and by interfering with MSC differentiation toward the osteoblastic phenotype [21]. Therefore, combination strategies combined proteasome inhibition with both vitamin D [22] and epigenetic regulators [23]. Building on these strategies, different groups unravelled novel mechanisms able to mobilize and eradicate nicheprotected myeloma cells by employing histone deacetylase inhibitors (HDACis) [24]. Pharmacological interfering with nucleosome conformation changes and skeletal metabolism demonstrated the interruption of the molecular crosstalk between MM cells and the stroma and uncovered indirect effects halting cell proliferation, bone disease, and angiogenesis, in vitro and in vivo [24-26].

The myeloma microenvironment is also characterized by Notch signalling hyperactivation due to the increased expression of Notch 1 and 2 and the ligands Jagged 1 and 2 in tumor cells. Notch activation influences myeloma cell biology and promotes the reprogramming of bone marrow stromal cells. Colombo et al. [27] uncovered Jagged blocking to be relevant for dismal sensitivity to alkylating agents, immunomodulatory drugs (IMiDs), and proteasomal 
inhibition due to $\mathrm{MM}$ cell and tumor milieu-related mechanisms. Enhanced CXCR4/SDF-1 alpha signalling is boosted by Notch overactivation within the MM environment. Additionally, this chemokine axis fuels antiapoptotic mechanisms [27], prompting therapeutic approaches holding the potential to interrupt the vicious cycle between the tumoral PCs and the BMSCs and, conceivably, improve patients' responses to standard-of-care therapies [27].

Furthermore, CXCR4/SDF-1 alpha signalling has been revealed to impact clinical outcome in PC dyscrasias. Nevertheless, treatment strategies pinpointing this receptor or its cognate ligand (burixafor or plerixafor) deemed not adequately proficient. Therefore, a deeper characterization of the biological CXCL12/CXCR4 interaction can offer additional insights, overcoming PC disorder treatment resistance and clonal resilience. This could allow envisioning a novel therapeutic window and a more effective drug combination, designed to halt myeloma progression [28, 29].

Additionally, glycosylation, by modulating different aspects of tumor biology, can be considered as a hallmark of cancer. Several solid and haematological malignancies are characterized by enhanced sialylated glycan expression, with a direct correlation with higher disseminated behaviour. Sialylation can also prime MM homing into its environment by physical interaction between skeletal precursors, stromal cells, and MM cells creating niches and educating bone cells. Therefore, interfering with sialylation may promote translational navigation of the milieu-drug resistance boundaries and define alternative combinatorial treatment strategies bringing sialylation inhibitors to the MM-stroma interface [30]. Thus, nanotechnologically engineered tools provided next-generation strategies for tailored anti-MM therapy by optimization of pharmacokinetics and pharmacodynamics profile of conventional chemotherapeutic agents [31]. In detail, novel anthracycline preparation integrating integrin $\alpha 4 \beta 1$ within nanoparticles seems to be able to exert enhanced anti-MM and dismal off-target effects, offering a proof of concept of the value of this pharmacokinetics innovation [31]. An alternative approach was delivering liposomal formulation carrying combinations of taxanes, alendronate, and isoform-adapted transferrin, enabling microenvironment drug modulation [32]. Notably, novel engineered tagging strategies combined modern immunotherapeutic targeting with either proteasome inhibitor [33] or bonemodifying agents (BMAs) [34], gaining more efficient offtarget profile. Specifically, CD38 receptor- and B-cell maturation antigen- (BCMA-) directed approaches have introduced a practice change in immunotherapy and are being intensively investigated in MM $[34,35]$, since these molecules are highly expressed on the malignant plasma cells.

Sialylation inhibition using these approaches also promises incremental activity in interfering within the MMniche vicious cycle. Recently, sialyltransferase inhibitors restored affective anti-MM activity by restoring innate and acquired immune response, while halting malignant cell proliferation at the same time [36].

Currently, delivery systems employing the sialylation inhibitor 3Fax-Neu5Ac encapsulation in combination with BMA are intensively investigated, in order to potentially block MM homing and enhance drug efficacy as well. In frame of this thinking, Natoni et al. [30] have shown that BMSCs can nurse MM by shaping an immune-tolerogenic milieu and uncovered sialylation as an actionable mechanism to boost the immune response [30].

2.1. Angiogenesis in Multiple Myeloma. In 1994, Vacca et al. [37] demonstrated for the first time that bone marrow microvascular density (MVD) was significantly increased in MM compared to monoclonal gammopathies of undetermined significance (MGUS) and even more in active vs. nonactive forms. The close association between angiogenesis and active MM indicates that it is the vascular phase of plasma cell tumors. Conversely, MGUS and nonactive MM represent the avascular phase. The microvessel area and the labeling index (LI) percent are closely associated with the MM activity phase and are mutually correlated [38-41].

In 2011, Ria and colleagues [15] also highlighted neovascularization as a constant hallmark of MM progression. This process is only partially supported by factors such as VEGF, fibroblast growth factor-2 (FGF-2), and metalloproteinases (MMPs), which are directly secreted by the tumor cells. As a consequence of plasma cell-stromal cell interactions, the cytokines within the MM niche, in particular IL-6, drive the release of angiogenic factors from bystanders in the bone milieu, these being one of the main triggers of the angiogenic switch during disease progression. But along with angiogenesis, vasculogenesis also occurs in the tumor niche of MM subjects, enhancing the vascular tree formation. In the neoplastic microenvironment of $\mathrm{MM}$ individuals, hematopoietic stem cells are primed to become ECs by the angiogenic cytokines shed in autocrine, paracrine, and endocrine fashion.

Therapeutic strategies in MM consist of conventional chemotherapy and biologically based therapy targeting not only MM-PCs but also the microenvironment and angiogenesis. Bortezomib regulates many cellular processes, including the modulation of transcription factors, such as NF$\kappa \mathrm{B}$, cell cycle progression, inflammation, immune surveillance, growth arrest, and apoptosis. NF- $\kappa \mathrm{B}$ is a major transcriptional factor which mediates the expression of many proteins including cytokines, chemokines, and cell adhesion molecules. Bortezomib inhibits NF- $\kappa \mathrm{B}$, enhancing the susceptibility of MM plasma cells to therapeutics, while the induction of IL- 6 by BMSCs mediated by NF- $\kappa$ B increases the secretion of VEGF from MM-PCs. Furthermore, bortezomib inhibits MMEC mitotic activity, through inhibition of VEGF, IL-6, insulin-like growth factor-1 (IGF-1), and angiopoietin-1 and angiopoietin-2 (Ang-1 and Ang-2) [42].

The circulating levels of Ang-1, Ang-2, VEGF, and angiogenin were measured in 54 patients with smouldering MM (SMM). This result was compared with those of 27 MGUS patients, $55 \mathrm{MM}$ patients, and 22 healthy controls, demonstrating that the ratio of circulating Ang-1/Ang-2 was reduced in MM individuals with full-blown overt MM due to a biologically significant enhancement of Ang-2, but not in SMM or MGUS nonmalignant control subjects. VEGF and 
angiogenin were increased in all patients compared to controls. However, circulating VEGF was higher in symptomatic MM compared to SMM and MGUS, while angiogenin was reduced. Hence, the above data show that the Ang-1/Ang-2/Tie-2 axis may be an effective target for the development of novel antimyeloma agents [43]. Bortezomib downregulates not only the caveolin-1 tyrosine phosphorylation, responsible for VEGF-mediated MM cell migration, but also the caveolin-1 phosphorylation induced by VEGF in ECs. Finally, bortezomib inhibits the transcription of ICAM1, VCAM1, and E-selectin [44].

Thalidomide is an antiangiogenic drug. It modulates tumor necrosis factor-alpha (TNF- $\alpha$ ) signalling through direct and indirect effects on the tumor microenvironment [45]. It also reduces FGF-2 [46], VEGF, and IL-6 secretion by BMSCs and MM cells [47], stimulating the activation and expansion of $T$ cells and enhancing NK-cell-mediated cytotoxicity. Thalidomide disrupts the host marrow-MM cell interactions by selectively modulating the density of cell surface adhesion molecules. Nonetheless, treatment with thalidomide induces side effects while lenalidomide and pomalidomide, its derivatives, are both less toxic and more potent [48, 49]. Cereblon (CRBN) is a primary target of thalidomide teratogenicity, but it is also required for the antimyeloma activity of thalidomide and related drugs (IMiDs). A decreased CRBN expression is linked to pharmacological resistance in human MM cell line models and primary cells and may also provide a biomarker to predict IMiD response and resistance [49]. In fact, other authors analysed the influence of the single-nucleotide polymorphisms (SNPs) of the CRBN gene on the risk of adverse effects of thalidomide-based chemotherapy in patients with MM [50].

However, Curry and colleagues found no reduction in MVD before or after treatment with thalidomide of newly diagnosed MM (NDMM) patients [51]. Nevertheless, other authors showed that high MVD at diagnosis was considered an independent poor prognosis factor [52].

Lamanuzzi and colleagues [53] evaluated mTOR activation in ECs from 20 patients with MGUS and 47 patients with $\mathrm{MM}$ and its involvement in angiogenesis. MTOR and the rapamycin-insensitive companion of mammalian target of rapamycin (RICTOR), two components of mTORC2 complex [54], were deemed significantly elevated in MMECs compared to MGUS-ECs. The authors uncovered mTORC2 expressed by MMECs to be relevant for angiogenic boosting and found that mTOR/RICTOR targeting by siRNA and dual mTOR inhibitor PP242 reduced the MMEC angiogenic functions, including cell migration, chemotaxis, adhesion, invasion, in vitro angiogenesis on Matrigel ${ }^{\circledR}$, and cytoskeleton reorganization. Additionally, in the chick embryo chorioallantoic membrane (CAM) and in Matrigel ${ }^{\circledR}$ plug assays, PP242-directed approaches demonstrated angiogenic blockade in vivo by interfering with angiogenesis. PP242 exerted a synergistic effect with IMiDs and proteasome inhibitor, suggesting that mTOR blockade can enhance the antiangiogenic effect of these drugs. Because mTORC2 involved in MM angiogenesis, dual mTOR inhibitor PP242 could support antiangiogenic management of MM patients [53].
Bisphosphonates exert a direct effect on MM plasma cells [55]. In detail, both zoledronic acid and neridronate have a cytotoxic activity on tumor cells and inhibit angiogenesis [55-57]. The side effect is osteonecrosis of the jaw (ONJ), a long-lasting disorder that occurs mainly in breast cancer and MM patients treated with intravenous bisphosphonates [58].

Recently, the role of CX3CL1/fractalkine has been reported, as a novel mechanism of this cell signalling boosting angiogenesis and inflammation in multiple myeloma. ADAM10 and ADAM17 are responsible for cleavage and shedding, thereby modulating CX3CL1/ fractalkine release. Notably, these MMPs are regulated by inflammatory and angiogenesis processes. Firstly, assessment of soluble levels in plasma cell disorders at different disease stages demonstrated that circulating concentration of CX3CL1 was significantly higher in fullblown disease compared with controls [59]. Strikingly, this observation was correlated with BM microvessel density. Next, ensuing functional in vitro experiments recapitulated fractalkine dynamics, highlighting the theragnostic role of enhanced production of this chemokine by MM-derived BM endothelium upon exogenous stimulus [59, 60]. In fact, Tanaka et al. [61] uncovered mAb-blocking strategies anti-CX3CL1 as nextgeneration approach aimed at halting innate and adaptive immune-dependent inflammation. Finally, Chen et al. also corroborated this evidence demonstrated decreased CX3CL1 production in vivo, upon proteasome inhibition [62]. These compelling data envision also the use of antiTNF- $\alpha$, in combination with the abovementioned therapeutic strategies for MM patients [59].

2.2. Endothelial Cells. MMECs express VEGF/VEGFR-2, FGF-2/FGF-2 receptor-2 (FGF-2R-2), and Ang-2/Tie-2 and exert an increased in vitro and in vivo angiogenic activity [63]. Moreover, MMECs express CXC chemokines CXCL8/ IL-8, CXCL11/interferon-inducible T-cell alpha chemoattractant (I-TAC), CXCL12/SDF- $1 \alpha$, and CCL2/monocyte chemotactic protein-1 (MCP-1), which mediate plasma cell proliferation and homing [64].

Long-term MMECs were compared with MGUSECs and HUVECs as their normal quiescent counterpart [63]. MMECs but not healthy cells overexpressed endothelial activation markers [65]. Mechanistically, MMECs increasingly produce FGF-2, VEGF, MMP-2, and MMP-9 compared to HUVECs, conferring a growth advantage over controls by a faster establishment of a proangiogenic phenotypic behavior, in terms of capillary sprouting and net formation [65]. MMECs also boost a strong proangiogenic response in the CAM [66]. Gene expression assays corroborated these pieces of evidence, uncovering MMECs' phenotype to be characterized by enhanced proangiogenic gene transcription, namely, VEGF, FGF isoforms, HGF, Tie2, transforming growth factor-beta (TGF- $\beta$ ), GRO- $\alpha$ chemokine, fibronectin-1, HIF-1 $\alpha$, ETS-1, ID3, and osteopontin compared to HUVECs. 
MMECs alone displayed a VEGF-dependent autocrine growth loop [65], owing to high VEGF and VEGFR-2 expression, constitutive autophosphorylation in both VEGFR2 and the associated kinase ERK-2, along with the inhibition of proliferation, capillarogenesis, and phosphorylation by neutralizing anti-VEGF and anti-VEGFR-2 antibodies. Pentraxin 3 affected MMEC functional activities and was able to modify the angiogenic capability of both MMECs and plasma cells [67].

Comparative gene expression profiling was made of MMECs and MGUSECs with Affymetrix U133A arrays [68]. Expression of 22 genes deemed significantly different by comparing MMECs with MGUSECs. Key biological processes related to protumorigenic functions were affected, showing significant gene expression deregulation in the symptomatic disease when compared to the precursor's states. Next, DIRAS3, SERPINF1, SRPX, BNIP3, $I E R 3$, and $S E P W 1$, gene-encoding proteins, were functionally tested to substantiate the gene signature findings, corroborating their proangiogenic function in BMECs. $B N I P 3, I E R 3$, and SEPW1 transient gene silencing had a significant impact on programmed death, cell proliferation, adhesion, and angiogenesis-related functions. Four proteins were found to be overexpressed in MMECs: filamin $\mathrm{A}$, vimentin, $\alpha$-crystallin $\mathrm{B}$, and $14-3-3 \zeta / \delta$ protein [69]. Their expression was enhanced by VEGF, FGF-2, HGF, and MM-PC-conditioned medium and their silencing RNA knockdown affected MMEC angiogenesisrelated functions, including spreading, migration, and tubular morphogenesis [69].

More recently, Leone described a novel aspect of disease pathophysiology, by characterizing the MM cell interface with the local environment, namely, vascular endothelium between ECs and $\mathrm{CD} 8^{+}$lymphocyte, create a permissive immune microenvironment within the BM, allowing plasma cell proliferation. In this context, the corrupted endothelium behaves as tolerogenic promoter, by indirect negative regulation of the effector memory $\mathrm{CD} 8^{+} \mathrm{T}$ cells. The $\mathrm{CD} 8^{+} \mathrm{T}$-cell population sustained by ECs also expressed Foxp3, producing IL-10 and TGF- $\beta$, and exerting a protumorigenic activity. The above study adds further insight into the role that ECs play in MM biology and describes an additional immune regulatory mechanism that inhibits the development of antitumor immunity and may impair the success of cancer immunotherapy [70].

Extracellular vesicles (EVs) shed from the MM cell surface actively participate in cellular crosstalk and vessel formation during MM progression [71]. Proteasome inhibition via Bi-EVs decreased EC proteasome activity, and BiEVs released from apoptotic MMECs promoted angiogenesis suppression by decreasing the proliferation and migration of ECs [71] IMiDs exerted a relevant antiangiogenic effect in vivo, and in vitro, it also inhibited migration of MMECs, but not of MGECs or control HUVECs. VEGF/ VEGFR-2 cell signalling was deemed biologically connected to lenalidomide treatment, which exerted a significant impact on cytoskeleton rearrangement, migration, and cell metabolism in MMECs [72].
2.3. Macrophages and Mast Cells. Tumor-associated macrophages and mast cells support tumor growth and neovascularization by producing a wide array of angiogenic cytokines. Mast cell- and macrophage-derived growth factors that can promote tumor development and angiogenesis include TNF- $\alpha$, TGF- $\beta 1$, FGF-2, VEGF, platelet-derived growth factor (PDGF), IL-8, osteopontin, and nerve growth factor (NGF). Conversely, mast cell- and macrophageproduced cytokines that may participate in antitumor responses include IL-1, IL-2, IL-4, IL-10, and interferongamma (IFN- $\gamma$ ) [73].

When BM macrophages from MM patients are exposed to VEGF and FGF-2, they transform into cells that are functionally and phenotypically similar to paired MMECs, and generate capillary-like networks mimicking those of MMECs [74]. Macrophages from nonactive MM, MGUS, and benign anaemia patients display similar albeit weaker features. EC-like macrophages and apparently typical macrophages contribute considerably to the formation of new vessels in patients with active MM, whereas their vascular supply is minimal in nonactive $\mathrm{MM}$ and absent in MGUS patients and control patients [74]. In contrast to MGUS and asymptomatic disease, CD14/CD68 surface overexpression has been found in full-blown myeloma. BM trephine immune staining additionally dissected two macrophage subpopulation, demonstrating cells with either endothelial or conventional phenotype by CD68/FVIII-RA coloration. Remarkably, proangiogenic effects on macrophages have shown to contribute to the building of neovessel wall in patients with active MM over nonactive and MGUS conditions [74].

Proinflammatory macrophages in BM biopsies are a potential prognostic biomarker for acquired MM resistance to bortezomib therapy, and high levels in BM are correlated with poor survival. Remarkably, proteasome inhibitor treatment of proinflammatory macrophages primed MMtumor-initiating cell (MM-TIC) infiltration both in vitro and in vivo in an IL- $1 \beta$-dependent fashion. One way to abolish bortezomib-induced MM-TIC enrichment is by blocking the IL1 $\beta$ axis using a pharmacologic or genetic approach [75]. Additionally, CD163 expression was detected by immunohistochemistry to determine the number of tumorassociated macrophages (TAMs) in $198 \mathrm{MM}$ patients receiving bortezomib-based regimens. Enhanced CD163+ TAM infiltration in NDMM was correlated with a worse clinical outcome, in terms of progression-free survival (PFS), overall survival (OS), and a worse therapy response quality compared with subjects with lower CD163+ TAM infiltration. These data indicate that the CD163+ TAM content at diagnosis is a powerful predictor of prognosis in MM [76]. Another link between the effect of bortezomib in MM patients and macrophages was highlighted by Khalife's studies. They demonstrated the improved treatment effectiveness gained by miR-16 increased expression in boosting anti-MM activity by a proteasome inhibitor in the presence of MM resident TAMs [77]. Enhanced soluble miR-16 in MM individuals linked to more favourable outcome. Conversely, deletion 13 on cancer cells was inversely associated with peripheral miR-16 concentration [78]. miR-16 can be 
actively secreted by MM cells through EVs, with a direct correlation between intracellular and shed levels. EVs isolated from MM patients can drive circulating monocyte differentiation to M2-TAMs, while the increased concentration of circulating miR-16 reverts this behaviour. In vivo, miR-16 lost sustains macrophage differentiation toward an M2 phenotype acquisition, most likely due to dismal NF- $\kappa$ B activation via IKK $\alpha / \beta$ targeting [77]. Moreover, the immune function of macrophages is mediated by IL-32 $\gamma$, which is overexpressed in MM patients and associated with a more advanced clinical stage [79]. Gene expression profiling showed a significant IL-32 $\gamma$-dependent induction of the immunosuppressive molecule indoleamine 2,3-dioxygenase (IDO) in macrophages, and this effect was verified by qRTPCR, western blotting, and immunofluorescence. Proteinase 3 (PR3), an IL-32 binding protein, was universally expressed on the surfaces of macrophages, and PR3 knockdown or the inhibition of the STAT3 and NF- $\kappa$ B pathways hindered the IL-32-gamma-mediated stimulation of IDO expression. These results indicate that MM cell-derived IL-32 $\gamma$ promotes the immunosuppressive function of macrophages and is a potential target for MM treatment [80].

MGUS and smouldering disease seem to be characterized by a peak of mast cell density count-related and angiogenesis enhancement [81]. Ang-1 is a crucial promoter of MM cell growth by stimulating angiogenesis. Experimental evidence indicates that Ang-1 secreted by primary murine mast cells promotes marked neovascularization in an in vivo transplantation assay [82]. Primary mast cells accelerate tumor growth by established plasmacytoma cell lines, while Ang-1-neutralizing antibodies significantly reduced the growth of plasmacytomas containing mast cells. Moreover, mast cell infiltrate parallels proangiogenic cytokine concentration, growth-related oncogene-alpha (GRO-alpha), and epithelial neutrophil-activating protein-78 (ENA-78). The authors also demonstrated that mast cell density was correlated with ki-67 PI, suggesting an important participation of mast cells in MM biology and growth [83]; in this context, mast cells would enhance angiogenesis, produce cytokines with growth effects on myeloma cells, and modify the BM microenvironment [84]. Therefore, mast cells could be indicators of the disease activity [85] and valuable targets for therapeutic interventions [83]. In line with this viewpoint, mast cells may be a novel target for an adjuvant strategy aimed at halting angiogenesis by interrupting the vicious cycle underlying cytotoxic cytokine production, thus circumventing mast cell-mediated immune suppression [86].

BM specimens from active myeloma over premalignant derived trephines analysed by both laser and electron microscopy were characterized by the presence of neovessels lined by granulated mast cells [87]. Otherwise, in MGUS, mast cells are localized on the abluminal side of neovessels [87]. However, mast cell density has an impact not only on angiogenesis but also on the progression of bone disease in MM patients. In $52 \mathrm{MM}$ patients, BM mast cell density was measured by immunohistochemical staining for tryptase, and serum levels of MMP-9 and RANKL were measured by a solid-phase sandwich enzyme-linked immunosorbent assay.
Additionally, urinary $\mathrm{N}$-terminal propeptide of procollagen type I (Ntx) concentrations was assessed by an enzymelinked assay, at different disease stages and bone involvement. Enhanced mast cell count, RANKL, and Ntx concentrations were found in MM subjects. Furthermore, mast cell density was positively correlated with MMP-9, RANKL, and Ntx. Therefore, mast cells may contribute to osteolytic processes during MM progression [88].

2.4. Cancer-Associated Fibroblasts (CAFs) in MM. In the progression of the disease from MGUS to MM, a fibroblast switch is required to acquire protumorigenic activity and parallels the behaviour exhibited by other haematological and solid cancers [89-92]. The switch was demonstrated by the bone marrow fibroblast gene expression profile of patients with MGUS and MM, extracted by nonnegative matrix factorization (NMF) [93]. Moreover, a specific miR profile in $\mathrm{BM}$ fibroblasts is linked to the transition from the asymptomatic to the full-blown disease. BM fibroblasts and EVdependent vicious cycle orchestrated by MM cells determine an enhanced production of miR-27b and miR-214, fuelling proliferative and antiapoptotic pathways. These prosurvival functions parallel an increased expression of fibroblast activation markers alpha-smooth muscle actin $(\alpha \mathrm{SMA})$ and fibroblast activation protein (FAP). While strengthening the mechanisms involved in the transition from MGUS and SMM to MM, a peculiar miRNA profile in MM-associated fibroblasts, along with the myeloma cells, educates the BM microenvironment by priming the BM fibroblast phenotype [94]. In fact, Desantis et al. studied the effect of recombinant human erythropoietin (rHuEPO) on MM fibroblasts in vivo and in vitro. It had previously been demonstrated that $\mathrm{rHuEPO}$ regulated angiogenic responses in $\mathrm{MM}$ via a direct effect on macrophages and ECs. Likewise, rHuEPO decreases the activation marker ( $\alpha \mathrm{SMA}$ and FAP) expression in MGUS and MM; furthermore, proinflammatory and proangiogenic cytokines, such as IL-6 and IL-8, VEGF-A, FGF-2, and HGF in MM fibroblasts, significantly diminished. Collectively, rHuEPO halted the MM-associated fibroblast proliferation. Conversely, fibroblast-programmed cell death enhanced in both MGUS and MM. Overall, these data pinpoint $\mathrm{rHuEPO}$ as a key brake on MM-supporting fibroblast action [95].

2.5. Myeloid-Derived Suppressor Cells. MDSCs are myeloid cells with a specific inhibitory activity on the immune response, which accumulate in the tumor microenvironment during tumor development [96] Significant accumulations of immunosuppressive MDSCs were observed in the BM of patients at early stages of MM and regulated MM growth by inhibiting T cells [97]. Moreover, murine MM cells directly activate BM MDSCs and enhance their immunosuppressive function through soluble factors such as granulocyte-macrophage colony-stimulating factor (GM-CSF), promoting the immune escape of MM cells [98]. An increase of bone marrow MDSCs was also detected in the 5T33 MM mouse model after inoculation with MM cells [98]. In the BM of 5T33 MM mice, exosomes derived from MM cells increased 
the number of BM MDSCs in vivo and induced changes in MDSC subpopulations which are similar to their phenotype, suggesting the involvement of exosomes in the accumulation of MDSCs [99].

MDSCs can mediate the suppression of myeloma-specific T-cell responses through the induction of T-cell anergy and Treg development in the MM microenvironment [100]. Polymorphonuclear (PMN)-MDSCs and neutrophils equivalently sustain $\mathrm{MM}$ resistance to alkylators and doxorubicin, by mediating soluble factor production. Targeting PMN-MDSCs could enhance chemotherapy efficacy in MM. It is well accepted that targeting MDSCs in cancer improves the immune response and increases the efficacy of immunotherapy. MDSCs play an ancillary role as a suitable target to overcome MM drug resistance, an important finding in light of recent data suggesting the benefit of combined chemo- and immunotherapy treatment protocols [100-102]. Due to the loss of equilibrium in the MM immune landscape, immune checkpoint targeting agents have not shown clinical activity in MM. It is therefore critically important to deal with immunosuppressive mechanisms and improve immune responses, especially in advanced MM patients. New immunotherapeutic strategies such as immunomodulatory drug-intensified monoclonal antibody treatment, immune checkpoint inhibitors, and chimeric antigen receptor T-cell therapy targeting B-cell maturation antigen have been employed in advanced-stage MM patients [103]. An association between high PMN-MDSC levels and poor overall survival in MM patients has been validated. PMN-MDSCs induced piRNA-823 upregulation, which in turn enhanced DNA methylation, thus stimulating the MM cell clonal evolution. Silencing of piRNA-823 in MM cells reduced the stemness of MSCs maintained by granulocytic (G)-MDSCs, resulting in a decreased tumor burden and angiogenesis in vivo [104]. It has also been demonstrated that the proinflammatory cytokine IL-18 is critically involved in MM and its levels are associated with MDSCs. IL18-deficient mice were remarkably protected from MM progression in a $\mathrm{CD} 8{ }^{+} \mathrm{T}$-cell-dependent manner. Within the BM milieu, IL-18 stimulates MDSCs, sustaining MM progression. High levels of BM plasma IL-18 were associated with poor survival in MM patients. The above preclinical studies suggested that IL-18 could be a potential therapeutic target in MM [105]. Additionally, the estrogen effect in hematologic malignancies including MM was studied, and treatment with 17 beta-estradiol significantly promoted the progression of the disease. However, this effect has not been attributed to a direct effect of estrogen on MM cells but was considered to be mediated through estrogen-induced alterations in the tumor microenvironment. In particular, it significantly increased the ability of MDSCs to suppress T-cell proliferation [106].

Botta et al. [107] pointed out developments in MDSCdirected approaches, by suggesting applications toward histone-deacetylase inhibitors in MM and uncovered signalling pathways involving MDSCs $[108,109]$, able to halt inflammation, by impairing JAK/STAT downstream. Epigenetic modulation reduced cell ability of monocytic phenotype granulocytic shift, by promoting macrophages or dendritic cells shaping within the tumor niche and opening novel therapeutic windows [110-112]. All-trans retinoic acid (ATRA) indeed enhanced MAPK activation with dismal reactive oxygen species levels, prompting mature myeloid lineage fuelling $[113,114]$.

Despite the existence of a correlation between MDSC pathophysiology and proangiogenic factors, VEGF-blocking $\mathrm{mAb}$ strategies did not succeed. Furthermore, the likelihood of MDSC-induced reduced sensitivity to the antiangiogenetic therapy discouraged further attempts in this direction [115]. Conversely, promising results generated by investigating miRNA-based approaches $[112,116]$ hold the potential to reduce MM disseminated potential and provided the bases to reveal MDSC-related targets to identify, mobilize, and eradicate niche-protected cells likely able to favor MM progression [117].

Aiming to implement MDSC-dependent immunosuppression halting strategies, several attempts have been made to interfere with cyclooxygenase-2 (COX-2), arginase-1 expression, and inducible nitric oxide synthases and to decrease reactive oxygen species production and provided undeniable rational for the novel association of anti-inflammatory compound to the MM therapeutic backbone, in order to expand the effectiveness of immunotherapy and to decrease the myeloid-derived population in the MM environment [118]. Remarkably, the antigen-presenting cell (APC) capacity of dendritic cells and ECs [76] can also open a further therapeutic window, since several examples have recently been published, highlighting the tight crosstalk between the immune microenvironment gene signature, vascular cells, and molecular targets, in both haematological and solid cancers $[119,120]$. These studies point to the WNT pathway as a druggable, theragnostic marker with a plethora of effects on the immune microenvironment in cancer [121].

This complex scenario fostered an intensive translational investigation aimed at improving $\mathrm{MM}$ immune equilibrium lost via MDSC targeting. Other noteworthy aspects are related to the immune microenvironmental landscape and its modulation through fluoropyrimidine, nucleoside analogues, and anthracyclines [122]. Nonetheless, state-of-theart development $[123,124]$ holds great potential in circumventing myeloid-derived immune suppression by interfering with critical signals, such as IL- 4 receptor $\alpha$ (IL$4 \mathrm{R} \alpha$ ), thus reducing cell proliferation. Moreover, the binding of the aptamer to its specific receptor led to MDSC depletion and tumor growth. Peptide enrichment in both $M-$ and G-MDSCs by phage-dependent strategies led to the development of a peptibody, via the fusion of peculiar peptide sequences with the $\mathrm{Fc}$ portion of murine $\mathrm{IgG} 2 \mathrm{~b}$ monoclonal antibodies, demonstrating in vivo activity [124].

The main complexities of the MM microenvironment cell network are summarized in Figure 1.

Multiple myeloma, MM; vascular endothelial growth factor, VEGF; nerve growth factor, NGF; fibroblast growth factor, FGF; interleukin, IL; tumor necrosis factor-alpha, TNF- $\alpha$; transforming growth factor-beta 1 , TGF- $\beta 1$; plateletderived growth factor, PDGF; hypoxia-inducible factor-1 alpha, HIF- $1 \alpha$; runt-related transcription factor, RUNXs; monocyte chemotactic protein 1, MCP 1; insulin-like growth factor 1, IGF-1; stromal cell-derived factor 1, SDF-1; MIP1; 


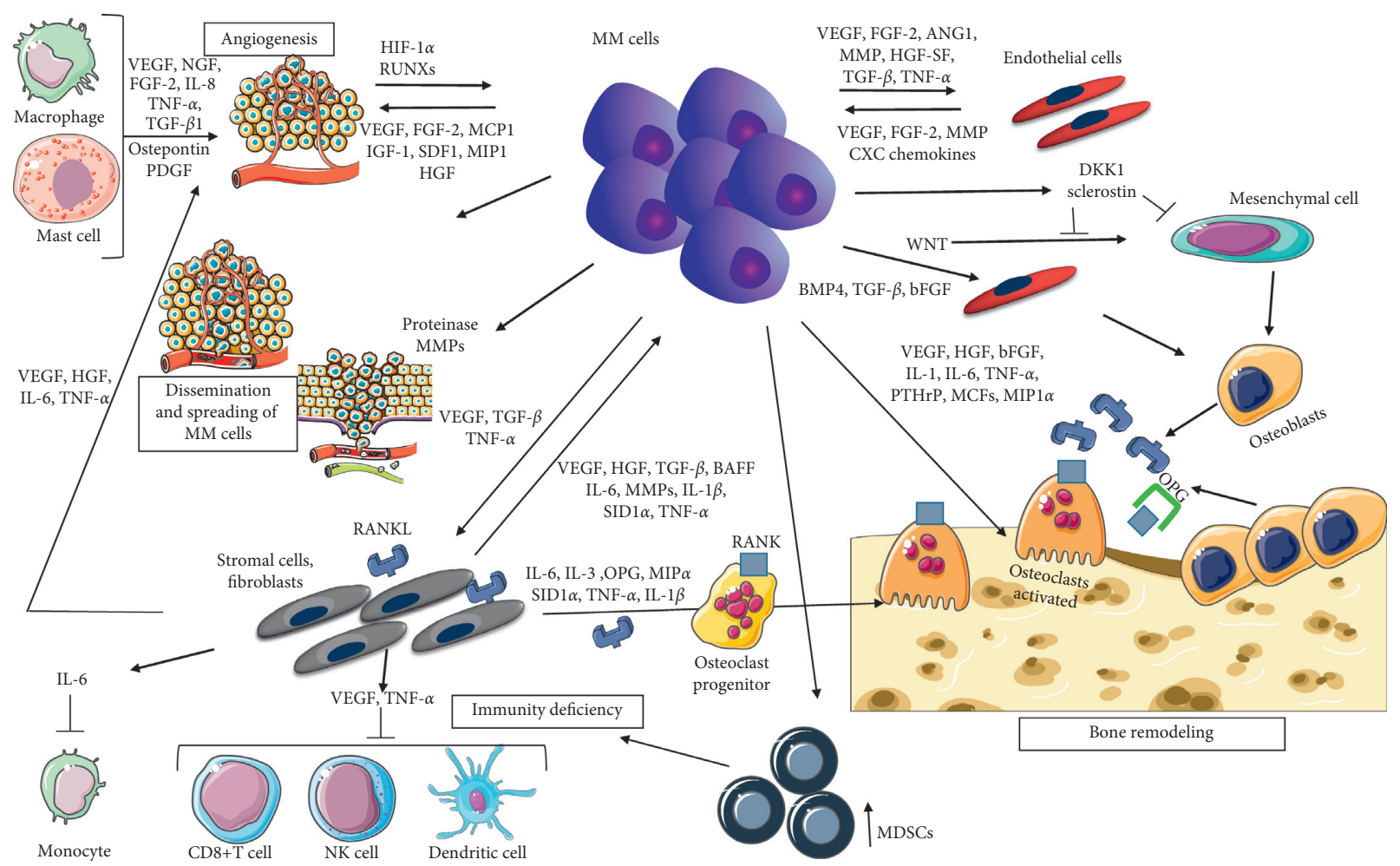

Figure 1: Soluble factors and adhesion-related interactions between MM cells and bone marrow immune microenvironment: MM-PCs prime the tumoral milieu via a plethora of mechanisms. Immune cell function, by deficiency in adaptive and innate response dysfunction, and proinflammatory cytokine production drive essential signals for microenvironment colonization and interactions. Moreover, MM-PCs control neoplastic survival and dormancy, modulating the response of the BM microenvironment cells to MM dissemination. Bone disease in $\mathrm{MM}$ is a prototypical malignant bone microenvironment pathologic condition. By tackling the knowledge gap on skeletal dissemination and disruption and cell-cell and cell-matrix interaction, the prevention and cure of MM progression may be better understood and targeted by immunomodulation, using combinations of MM-PC-directed agents against novel therapeutic targets.

angiopoietin 1, ANG1; metalloproteinases, MMP; hepatocyte growth factor/scatter factor (HGF/SF), chemokine, CXC; Dickkopf, DKK; wingless-type MMTV integration site family, WNT; bone morphogenetic protein-4, BMP4; parathyroid hormone-related protein, PTHrP; macrophage colony-stimulating factors, MCFs; B-cell activating factor, BAFF; SID1 transmembrane family, member 1, SID1; receptor activator of nuclear factor-kappa-B ligand, RANKL; osteoprotegerin, OPG; natural killer, NK; myeloid-derived suppressor cells, MDSCs.

\section{Therapeutic Windows}

A new goal in haematological malignancies is represented by a treatment approach targeting not only patients with active MM, but also those with SMM. This shift toward early intervention [125] with the antiangiogenic agents lenalidomide and dexamethasone demonstrated prolonged disease-free survival and OS in patients with SMM.

\section{Targeting Angiogenesis and the Immune Microenvironment in Multiple Myeloma: Current Challenges}

The knowledge of critical pathways supporting angiogenesis and creating immunosuppression during MM evolution uncovered a reciprocal crosstalk between MM cells with the surrounding milieu, and compelling verification designates that angiogenesis and immunosuppression often fuel a simultaneous vicious cycle [70]. Consequently, approaches relating to antiangiogenic immune mechanisms seem to hold the promise to tip the equilibrium of the MM environment and increase clinical benefit. The first-in-class drug thalidomide and its derivative lenalidomide mirrored the abovementioned knowledge, representing one possible translation from bench-tobedside efforts [51, 126]. But as stated above, the precise target of lenalidomide is cereblon, since low cereblon levels are associated with drug resistance [127-129]. Undeniable evidence supports the use of drugs that target the BM microenvironment to prevent the progression of SMM or full-blown MM. Additionally, in a mouse model, the use of an antiangiogenic anti-VEGFR-2 antibody in the early stage delayed tumor progression of MM; nonetheless, besides IMiDs, angiogenic-directed strategy did not show effective results in the unselected patient subgroup in patients with MM [130]. In order to achieve a patienttailored vasculogenic targeting, stringent patient stratification has been proposed by modulating from the critical step of MM evolution that can be critically dependent on vessel supply, such as smouldering phases [131] or extramedullary dissemination [132-134]. 
TABLE 1: Compounds in advanced investigation targeting MM cells and the tumor microenvironment.

\begin{tabular}{|c|c|}
\hline Targets & Therapeutic agents \\
\hline Angiogenesis & $\begin{array}{l}\text { (1) IMiDs (thalidomide*, lenalidomide* }) \\
\text { (2) Anti-VEGF Ab (bevacizumab) } \\
\text { (3) Tyrosine kinase inhibitor against VEGF (sunitinib) }\end{array}$ \\
\hline Hypoxia & (1) Evofosfamide investigational hypoxia-activated prodrug \\
\hline Stromal cells & $\begin{array}{l}\text { (1) CXCR4 inhibitors (plerixafor) } \\
\text { (2) Anti-CXCR4 Ab (ulocuplumab) } \\
\text { (3) CXCL12 inhibitor (olaptesed pegol) }\end{array}$ \\
\hline Osteoclasts and osteoblast & $\begin{array}{l}\text { (1) Bisphosphonates* } \\
\text { (2) Anti-DKK1 Ab } \\
\text { (3) Anti-RANKL Ab (denosumab*) } \\
\text { (4) Antisclerostin mAb }\end{array}$ \\
\hline Cytokine signalling & $\begin{array}{l}\text { (1) TGF- } \beta \text { antagonists (sotatercept) } \\
\text { (2) TNF- } \alpha \text { antagonists (etanercept) } \\
\text { (3) Anti-TNF- } \alpha \text { Ab (infliximab) } \\
\text { (4) IL-6 antagonist (siltuximab) }\end{array}$ \\
\hline Stimulation of anticancer immunity & $\begin{array}{l}\text { (1) Anti-PD-1/PD-L1 Ab (pembrolizumab, nivolumab) } \\
\text { (2) Anti-CTLA4 Ab (ipilimumab) } \\
\text { (3) CAR-T cells } \\
\text { (4) MILs } \\
\text { (5) Vaccines } \\
\text { (6) Anti-CD38 Ab (daratumumab*, isatuximab) } \\
\text { (7) Anti-SLAMF7 Ab (elotuzumab*) }\end{array}$ \\
\hline
\end{tabular}

Immunomodulatory drugs, IMiDs; vascular endothelial growth factor, VEGF; antibody, Ab; chemokine receptor 4, CCR4; chemokine ligand 12, CXCL12; Dickkopf, DKK; receptor activator of nuclear factor-kappa-B ligand, RANKL; transforming growth factor-beta 1, TGF- $\beta 1$; tumor necrosis factor-alpha, TNF$\alpha$; interleukin-6, IL-6; programmed cell death 1/programmed cell death ligand 1, PD-1/PD-L1; chimeric antigen receptor-T cells, CAR-T cells; marrow infiltrating lymphocytes, MILs; self-ligand receptor of the signalling lymphocytic activation molecule, SLAMF7. *FDA and EMA approved.

The next breakthrough of therapeutic strategy design is targeting the MM ecosystem together with the immune microenvironment. The altered BM niche sustains the proliferation of MM cells, nursed by physical and soluble reciprocal interactions educating both the neoplastic and the immune environmental cells [135]. Identification, mobilization, and eradication of this niche-protected dormant and often pharmacological insensitive cells have been significantly improved since several trials involving antibodies have proved clinical benefits in MM. Anti-SLAMF7 targeting by elotuzumab anti-CD38-directed approaches by daratumumab as a single agent or with proteasome inhibitor and IMiDs have shown far more effectiveness and superior activity than the standard of care [136, 137]. The first-in-class SLAMF7 targeting molecule stimulates NK cells and macrophages; conversely, CD38-targeting by daratumumab induces the immune system triggering toward Treg reduction and by enhancing T-helper and cytotoxic lymphocytes [138]. Another target studied especially in melanoma, lung cancer, and Hodgkin lymphoma is programmed cell death 1 (PD-1) $[139,140]$. In more detail, the pieces of evidence that PD-1/ PD-ligand 1 (PD-L1) modulation increases T- and NK cell antimyeloma effects prompted the use of immune checkpoint inhibitors in clinical studies. Nevertheless, the anti-PD-1/PD$\mathrm{L} 1 \mathrm{mAb}$ as a single agent did not provide sufficient results. Lack of infiltrating effector cells within the MM milieu can explain the modest efficacy demonstrated by these clinical trials [141]. Therefore, drug combination strategies encouraged clinical trials (NCT02289222 and NCT02331368), uncovering encouraging medical response $[142,143]$. In addition, studies of chimeric antigen receptor-T-cell (CAR-Tcell) therapy targeting BCMA have revealed very high response rates in heavily pretreated patients with $\mathrm{MM}$ $[140,144]$.

As pointed out, CXCL12 and its ligand CXCRA can have a pathologic role in different stages of $\mathrm{MM}$ and patient drug resistance, so disrupting the CXCL12-CXCR4 axis might be a therapeutic opportunity [39]. Roccaro et al. uncovered CXCL12 and CXCR4 as putative targets to halt MM evolution and extramedullary dissemination in animal models [28, 145], indicating broad potential consequences on adhesion-mediated MM dissemination $[29,101,146]$ and drug resistance, as in other solid and haematological malignancies [147-149] and prompt clinical validation [150]. Therapeutic interventions with burixafor or plerixafor (CXCR4 antagonists) in MM are not efficient enough [39].

Bisphosphonates (pamidronate or zoledronic acid) [56] and other BMAs, such as DKK1 inhibitors (Dickkopf WNT signalling pathway inhibitors), antisclerostin $\mathrm{mAb}$ [151], and RANKL inhibitors (denosumab) [152], represent an example of attempts to target the disseminated and localized bone disease effect due to the spreading of malignant plasma cells. Nonetheless, despite encouraging clinical outcome gained in full-blown disease [153], to date no clinical evidence demonstrated a survival benefit by treating the asymptomatic version of myeloma [154].

Studies conducted on MM cell lines have shown that the addition of exogenous IL- 6 is essential for obtaining the growth of neoplastic cells in vitro. By removing IL-6 from short-term cultures, MM cells die, demonstrating that this 


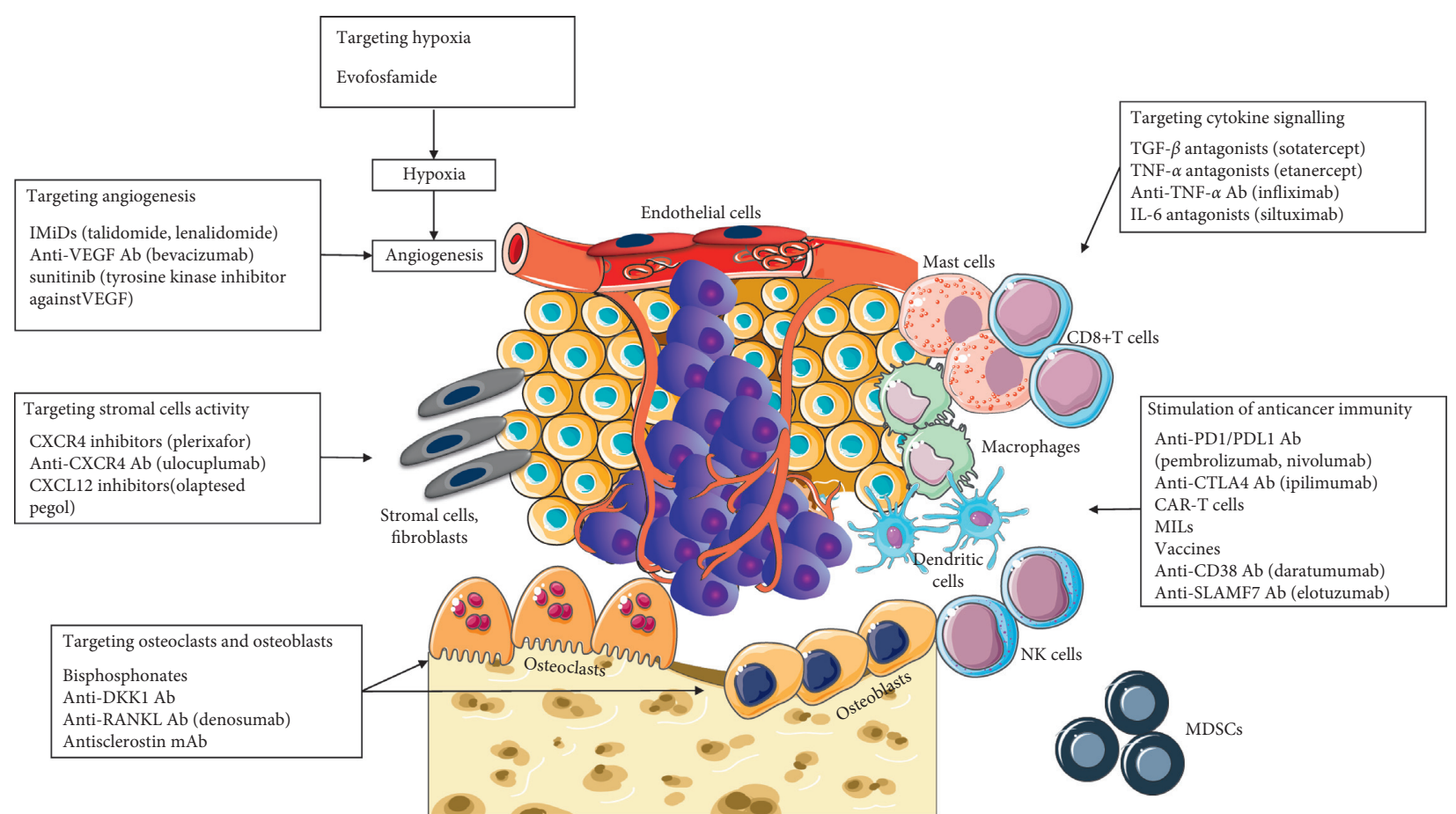

FIgURE 2: Therapeutic targets within the tumor milieu in multiple myeloma. Immunomodulatory drugs, IMiDs; vascular endothelial growth factor, VEGF; antibody, Ab; chemokine receptor 4, CCR4; chemokine ligand 12, CXCL12; Dickkopf, DKK; receptor activator of nuclear factor-kappa-B ligand, RANKL; transforming growth factor-beta 1 , TGF- $\beta 1$; tumor necrosis factor-alpha, TNF- $\alpha$; interleukin-6, IL-6; programmed cell death 1/programmed cell death ligand 1, PD-1/PD-L1; chimeric antigen receptor-T cells, CAR-T cells; marrow infiltrating lymphocytes, MILs; self-ligand receptor of the signalling lymphocytic activation molecule, SLAMF7.

cytokine acts as both a growth factor and a survival factor. In long-term and high cell density cultures, malignant plasma cells become progressively independent and are able to produce IL-6 as an autocrine growth factor [155]. These pieces of evidence prompted several investigations aimed at characterizing additional soluble substances supporting MM in the environment. TGF- $\beta$ is one of the best candidates deserving druggable intervention investigation [156], employing several blocking approaches [157, 158], demonstrating clinical activity in the treatment of MM-associated bone diseases. Luspatercept treatment has been shown to have a potential impact on MM-related kidney involvement (Table 1 and Figure 2).

Ultimately, adequate oxygen pressure is essential for proper physiologic conditions and insufficient hypoxia is a conspicuous characteristic in various physiological and pathological processes, including neoplastic disorders and cancer dissemination [159]. In MM, increased BM hypoxia is associated with an increased recirculation of neoplastic cells [160], leading to loss of pharmacological sensitivity and priming resistance to radiotherapy [161]. Therefore, alkylators selectively activated by insufficient oxygen supply condition were tested in preclinical models [162] and in clinical trials involving patients with MM $[162,163]$, showing an effective inhibition of HIF-1-alpha in MM both alone and in combination [164]. Intriguing results from phase I/II clinical studies results supported further investigation in relapsed/refractory subjects [163]. The therapeutic opportunity window and pathophysiological aspects are summarized in Figure 2.

\section{Conclusions}

MM is likely one of the hematologic conditions in which the major advances from biology to new therapy have occurred over the last years. The biology outlook has shifted from morphology and basic biochemical analysis to an integrated multi-Omics approach offering novel therapeutic perspectives. Nonetheless, MM finally progresses to a relapse/refractory stage, levying a heavy impact on patient survival and quality of life. The MM microenvironment pathophysiologic determinants, defined from a validated prognostic perspective, provide clinicians with novel insight, offering the potential to deal with the unmet medical need for prolonged, sustained disease remission. The interactions of multiple myeloma cells with different subsets of immune cells and ECs within the BM tumor niche environment seem to be the ideal backbone supporting the ultimate translation of biological findings into improved diagnostics and therapies.

\section{Conflicts of Interest}

The authors declare that they have no conflicts of interest.

\section{Acknowledgments}

The authors disclose the support received by the Italian Association for Cancer Research (AIRC) through an Investigator Grant (no. 20441), by the GLOBALDOC Project to AGS. This research project was supported in part by the 
Apulian Regional Project "Medicina di Precisione" to AGS. The authors thank SMART (Servier Medical Art, http:// smart.servier.com/) for providing comprehensive medical and biological figures and fruitful datasets for the international scientific community. The authors also thank Mary Victoria Pragnell, BA, from the University of Bari Medical School for linguistic revision.

\section{References}

[1] P. Moreau, J. San Miguel, P. Sonneveld et al., "Multiple myeloma: ESMO clinical practice guidelines for diagnosis, treatment and follow-up," Annals of Oncology, vol. 28, pp. 52-61, 2017.

[2] S. K. Kumar, A. Dispenzieri, M. Q. Lacy et al., "Continued improvement in survival in multiple myeloma: changes in early mortality and outcomes in older patients," Leukemia, vol. 28, no. 5, pp. 1122-1128, 2014.

[3] B. G. M. Durie and S. E. Salmon, "A clinical staging system for multiple myeloma correlation of measured myeloma cell mass with presenting clinical features, response to treatment, and survival," Cancer, vol. 36, no. 3, pp. 842-854, 1975.

[4] P. R. Greipp, J. S. Miguel, B. G. M. Durie et al., "International staging system for multiple myeloma," Journal of Clinical Oncology, vol. 23, no. 15, pp. 3412-3420, 2005.

[5] A. Palumbo, H. Avet-Loiseau, S. Oliva et al., "Revised international staging system for multiple myeloma: a report from international myeloma working group," Journal of Clinical Oncology, vol. 33, no. 26, pp. 2863-2869, 2015.

[6] N. Bolli, G. Biancon, M. Moarii et al., "Analysis of the genomic landscape of multiple myeloma highlights novel prognostic markers and disease subgroups," Leukemia, vol. 32, no. 12, pp. 2604-2616, 2018.

[7] C. Pawlyn and G. J. Morgan, "Evolutionary biology of highrisk multiple myeloma," Nature Reviews Cancer, vol. 17, no. 9, pp. 543-556, 2017.

[8] N. J. Bahlis, "Darwinian evolution and tiding clones in multiple myeloma," Blood, vol. 120, no. 5, pp. 927-928, 2012.

[9] S. Manier, A. Sacco, X. Leleu, I. M. Ghobrial, and A. M. Roccaro, "Bone marrow microenvironment in multiple myeloma progression," Journal of Biomedicine and Biotechnology, vol. 2012, Article ID 157496, 2012.

[10] Y. Kawano, M. Moschetta, S. Manier et al., "Targeting the bone marrow microenvironment in multiple myeloma," Immunological Reviews, vol. 263, no. 1, pp. 160-172, 2015.

[11] I. M. Ghobrial, A. Detappe, K. C. Anderson, and D. P. Steensma, "The bone-marrow niche in MDS and MGUS: implications for AML and MM," Nature Reviews Clinical Oncology, vol. 15, no. 4, pp. 219-233, 2018.

[12] D. Ribatti, B. Nico, and A. Vacca, "Importance of the bone marrow microenvironment in inducing the angiogenic response in multiple myeloma," Oncogene, vol. 25, no. 31, pp. 4257-4266, 2006.

[13] M. Lemaire, S. Deleu, E. De Bruyne, E. Van Valckenborgh, E. Menu, and K. Vanderkerken, "The microenvironment and molecular biology of the multiple myeloma tumor," Advances in Cancer Research, vol. 110, pp. 19-42, 2011.

[14] I. R. Ramachandran, T. Condamine, C. Lin et al., "Bone marrow PMN-MDSCs and neutrophils are functionally similar in protection of multiple myeloma from chemotherapy," Cancer Letters, vol. 371, no. 1, pp. 117-124, 2016.

[15] R. Ria, A. Reale, A. De Luisi, A. Ferrucci, M. Moschetta, and A. Vacca, "Bone marrow angiogenesis and progression in multiple myeloma," American Journal of Blood Research, vol. 1, no. 1, pp. 76-89, 2011.

[16] T. Harding, L. Baughn, S. Kumar, and B. Van Ness, "The future of myeloma precision medicine: integrating the compendium of known drug resistance mechanisms with emerging tumor profiling technologies," Leukemia, vol. 33, no. 4, pp. 863-883, 2019.

[17] K. Vandyke, M. N. Zeissig, D. R. Hewett et al., "HIF-2 $\alpha$ promotes dissemination of plasma cells in multiple myeloma by regulating CXCL12/CXCR4 and CCR1," Cancer Research, vol. 77, no. 20, pp. 5452-5463, 2017.

[18] S. Xu, K. De Veirman, A. De Becker, K. Vanderkerken, and I. Van Riet, "Mesenchymal stem cells in multiple myeloma: a therapeutical tool or target?" Leukemia, vol. 32, no. 7, pp. 1500-1514, 2018.

[19] N. Rabin, C. Kyriakou, L. Coulton et al., "A new xenograft model of myeloma bone disease demonstrating the efficacy of human mesenchymal stem cells expressing osteoprotegerin by lentiviral gene transfer," Leukemia, vol. 21, no. 10, pp. 2181-2191, 2007.

[20] S. Ciavarella, G. Grisendi, M. Dominici et al., "In vitro antimyeloma activity of TRAIL-expressing adipose-derived mesenchymal stem cells," British Journal of Haematology, vol. 157, no. 5, pp. 586-598, 2012.

[21] S. Mukherjee, N. Raje, J. A. Schoonmaker et al., "Pharmacologic targeting of a stem/progenitor population in vivo is associated with enhanced bone regeneration in mice," The Journal of Clinical Investigation, vol. 118, no. 118, pp. 491504, 2008.

[22] M. F. Kaiser, U. Heider, M. Mieth, C. Zang, I. von Metzler, and O. Sezer, "The proteasome inhibitor bortezomib stimulates osteoblastic differentiation of human osteoblast precursors via upregulation of vitamin D receptor signalling," European Journal of Haematology, vol. 90, no. 4, pp. 263-272, 2013.

[23] S. Xu, K. De Veirman, H. Evans et al., "Effect of the HDAC inhibitor vorinostat on the osteogenic differentiation of mesenchymal stem cells in vitro and bone formation in vivo," Acta Pharmacologica Sinica, vol. 34, no. 5, pp. 699709, 2013.

[24] M. Ho, T. Chen, J. Liu et al., "Targeting histone deacetylase 3 (HDAC3) in the bone marrow microenvironment inhibits multiple myeloma proliferation by modulating exosomes and IL-6 trans-signaling," Leukemia, vol. 34, no. 1, pp. 196-209, 2019.

[25] T. J. Heino, J. J. Alm, H. J. Halkosaari, and V.-V. Välimäki, "Zoledronic acid in vivo increases in vitro proliferation of rat mesenchymal stromal cells," Acta Orthopaedica, vol. 87, no. 4, pp. 412-417, 2016.

[26] L. Hu, Y. Wen, J. Xu et al., "Pretreatment with bisphosphonate enhances osteogenesis of bone marrow mesenchymal stem cells," Stem Cells and Development, vol. 26, no. 2, pp. 123-132, 2017.

[27] M. Colombo, S. Garavelli, M. Mazzola et al., "Multiple myeloma exploits Jagged 1 and Jagged 2 to promote intrinsic and bone marrow-dependent drug resistance," Haematologica, 2019.

[28] A. M. Roccaro, Y. Mishima, A. Sacco et al., "CXCR4 regulates extra-medullary myeloma through epithelial-mesenchymaltransition-like transcriptional activation," Cell Reports, vol. 12, no. 4, pp. 622-635, 2015.

[29] T. R. Ullah, "The role of CXCR4 in multiple myeloma: cells' journey from bone marrow to beyond," Journal of Bone Oncology, vol. 17, Article ID 100253, 2019. 
[30] A. Natoni, R. Bohara, A. Pandit, and M. O’Dwyer, “Targeted approaches to inhibit sialylation of multiple myeloma in the bone marrow microenvironment," Frontiers in Bioengineering and Biotechnology, vol. 7, p. 252, 2019.

[31] T. Kiziltepe, J. D. Ashley, J. F. Stefanick et al., "Rationally engineered nanoparticles target multiple myeloma cells, overcome cell-adhesion-mediated drug resistance, and show enhanced efficacy in vivo," Blood Cancer Journal, vol. 2, no. 4, p. e64, 2012.

[32] Q. Chang, R. Geng, S. Wang, D. Qu, and X. Kong, "DOPAbased paclitaxel-loaded liposomes with modifications of transferrin and alendronate for bone and myeloma targeting," Drug Delivery, vol. 23, no. 9, pp. 3629-3638, 2016.

[33] P. de la Puente, M. J. Luderer, C. Federico et al., "Enhancing proteasome-inhibitory activity and specificity of bortezomib by CD38 targeted nanoparticles in multiple myeloma," Journal of Controlled Release, vol. 270, pp. 158-176, 2018.

[34] A. Swami, M. R. Reagan, P. Basto et al., "Engineered nanomedicine for myeloma and bone microenvironment targeting," Proceedings of the National Academy of Sciences, vol. 111, no. 28, pp. 10287-10292, 2014.

[35] N. W. C. J. van de Donk and S. Z. Usmani, "CD38 antibodies in multiple myeloma: mechanisms of action and modes of resistance," Frontiers in Immunology, vol. 9, p. 2134, 2018.

[36] C. Büll, T. J. Boltje, N. Balneger et al., "Sialic acid blockade suppresses tumor growth by enhancing T-cell-mediated tumor immunity," Cancer Research, vol. 78, no. 78, pp. 3574-3588, 2018.

[37] A. Vacca, D. Ribatti, L. Roncali et al., "Bone marrow angiogenesis and progression in multiple myeloma," British Journal of Haematology, vol. 87, no. 3, pp. 503-508, 1994.

[38] S. Schreiber, J. Ackermann, A. Obermair et al., "Multiple myeloma with deletion of chromosome $13 q$ is characterized by increased bone marrow neovascularization," British Journal of Haematology, vol. 110, no. 3, pp. 605-609, 2000.

[39] S. V. Rajkumar, S. Hayman, M. A. Gertz et al., "Combination therapy with thalidomide plus dexamethasone for newly diagnosed myeloma," Journal of Clinical Oncology, vol. 20, no. 21, pp. 4319-4323, 2002.

[40] G. Pruneri, M. Ponzoni, A. J. M. Ferreri et al., "Microvessel density, a surrogate marker of angiogenesis, is significantly related to survival in multiple myeloma patients," British Journal of Haematology, vol. 118, no. 3, pp. 817-820, 2002.

[41] M. G. Alexandrakis, F. J. Passam, E. Ganotakis et al., "Bone marrow microvascular density and angiogenic growth factors in multiple myeloma," Clinical Chemistry and Laboratory Medicine (CCLM), vol. 42, pp. 1122-1126, 2004.

[42] A. Roccaro, T. Hideshima, P. Richardson et al., "Bortezomib as an antitumor agent," Current Pharmaceutical Biotechnology, vol. 7, no. 6, pp. 441-448, 2006.

[43] M. Gkotzamanidou, D. Christoulas, V. L. Souliotis, A. Papatheodorou, M. A. Dimopoulos, and E. Terpos, "Angiogenic cytokines profile in smoldering multiple myeloma: no difference compared to MGUS but altered compared to symptomatic myeloma," Medical Science Monitor, vol. 19, pp. 1188-1194, 2013.

[44] F. Pajonk and W. H. McBride, "The proteasome in cancer biology and treatment," Radiation Research, vol. 156, no. 5, pp. 447-459, 2001.

[45] C. M. Segeren, P. Sonneveld, B. van der Holt et al., "Overall and event-free survival are not improved by the use of myeloablative therapy following intensified chemotherapy in previously untreated patients with multiple myeloma: a prospective randomized phase 3 study," Blood, vol. 101, no. 6, pp. 2144-2151, 2003.

[46] R. J. D’Amato, M. S. Loughnan, E. Flynn, and J. Folkman, "Thalidomide is an inhibitor of angiogenesis," Proceedings of the National Academy of Sciences, vol. 91, no. 9, pp. 40824085, 1994.

[47] D. Ribatti and A. Vacca, "Novel therapeutic approaches targeting vascular endothelial growth factor and its receptors in haematological malignancies," Current Cancer Drug Targets, vol. 5, no. 8, pp. 573-578, 2005.

[48] R. García-Sanz, J. R. González-Porras, J. M. Hernández et al., "The oral combination of thalidomide, cyclophosphamide and dexamethasone (ThaCyDex) is effective in relapsed/ refractory multiple myeloma," Leukemia, vol. 18, no. 4, pp. 856-863, 2004.

[49] Y. X. Zhu, K. M. Kortuem, and A. K. Stewart, "Molecular mechanism of action of immune-modulatory drugs thalidomide, lenalidomide and pomalidomide in multiple myeloma," Leukemia \& Lymphoma, vol. 54, no. 4, pp. 683-687, 2013.

[50] R. Mlak, A. Szudy-Szczyrek, M. Mazurek et al., "Polymorphisms in the promotor region of the CRBN gene as a predictive factor for peripheral neuropathy in the course of thalidomide-based chemotherapy in multiple myeloma patients," British Journal of Haematology, vol. 186, no. 5, pp. 695-705, 2019.

[51] P. C. de Camargo Cury, F. Higashi, F. F. S. Zacchi et al., "Effect of thalidomide on bone marrow angiogenesis in multiple myeloma patients," Hematology, Transfusion and Cell Therapy, 2019.

[52] N. Lee, H. Lee, S. Y. Moon et al., "Adverse prognostic impact of bone marrow microvessel density in multiple myeloma," Annals of Laboratory Medicine, vol. 35, no. 6, pp. 563-569, 2015.

[53] A. Lamanuzzi, I. Saltarella, V. Desantis et al., "Inhibition of mTOR complex 2 restrains tumor angiogenesis in multiple myeloma," Oncotarget, vol. 9, pp. 20563-20577, 2018.

[54] R. A. Saxton and D. M. Sabatini, "mTOR signaling in growth, metabolism, and disease," Cell, vol. 168, no. 6, pp. 960-976, 2017.

[55] C. Scavelli, G. Di Pietro, T. Cirulli et al., "Zoledronic acid affects over-angiogenic phenotype of endothelial cells in patients with multiple myeloma," Molecular Cancer Therapeutics, vol. 6, no. 12, pp. 3256-3262, 2007.

[56] P. Zardin, "The antitumor potential of bisphosphonates," Seminars in Oncology, vol. 29, no. 6, pp. 33-42, 2002.

[57] D. Ribatti, B. Nico, D. Mangieri et al., "Neridronate inhibits angiogenesis in vitro and in vivo," Clinical Rheumatology, vol. 26, no. 7, pp. 1094-1098, 2007.

[58] A. O. Hoff, B. B. Toth, K. Altundag et al., "Frequency and risk factors associated with osteonecrosis of the jaw in cancer patients treated with intravenous bisphosphonates," Journal of Bone and Mineral Research, vol. 23, no. 6, pp. 826-836, 2008.

[59] V. Marchica, D. Toscani, A. Corcione et al., "Bone marrow CX3CL1/fractalkine is a new player of the pro-angiogenic microenvironment in multiple myeloma patients," Cancers, vol. 11, 2019.

[60] A. G. Solimando, M. C. Da Vià, S. Cicco et al., "High-risk multiple myeloma: integrated clinical and omics approach dissects the neoplastic clone and the tumor micro environment," Journal of Clinical Medicine, vol. 8, no. 7, 2019.

[61] Y. Tanaka, T. Takeuchi, H. Umehara et al., "Safety, pharmacokinetics, and efficacy of E6011, an antifractalkine 
monoclonal antibody, in a first-in-patient phase $1 / 2$ study on rheumatoid arthritis," Modern Rheumatology, vol. 28, no. 1, pp. 58-65, 2018.

[62] F.-T. Chen, Y.-C. Liu, C.-M. Yang, and C.-H. Yang, "Antiinflammatory effect of the proteasome inhibitor bortezomib on endotoxin-induced uveitis in rats," Investigative Opthalmology \& Visual Science, vol. 53, no. 7, pp. 3682-3694, 2012.

[63] A. Vacca, R. Ria, F. Semeraro et al., "Endothelial cells in the bone marrow of patients with multiple myeloma," Blood, vol. 102, no. 9, pp. 3340-3348, 2003.

[64] A. Pellegrino, R. Ria, G. D. Pietro et al., "Bone marrow endothelial cells in multiple myeloma secrete CXC-chemokines that mediate interactions with plasma cells," British Journal of Haematology, vol. 129, no. 2, pp. 248-256, 2005.

[65] R. Ria, F. Russo, T. Cirulli et al., "A VEGF-dependent autocrine loop mediates proliferation and capillarogenesis in bone marrow endothelial cells of patients with multiple myeloma," Thrombosis and Haemostasis, vol. 92, no. 12, pp. 1438-1445, 2004.

[66] D. Ribatti, G. De Falco, B. Nico, R. Ria, E. Crivellato, and A. Vacca, "In vivo time-course of the angiogenic response induced by multiple myeloma plasma cells in the chick embryo chorioallantoic membrane," Journal of Anatomy, vol. 203, no. 3, pp. 323-328, 2003.

[67] A. Basile, M. Moschetta, P. Ditonno et al., "Pentraxin 3 (PTX3) inhibits plasma cell/stromal cell cross-talk in the bone marrow of multiple myeloma patients," The Journal of Pathology, vol. 229, no. 1, pp. 87-98, 2013.

[68] R. Ria, K. Todoerti, S. Berardi et al., "Gene expression profiling of bone marrow endothelial cells in patients with multiple myeloma," Clinical Cancer Research, vol. 15, no. 17, pp. 5369-5378, 2009.

[69] S. Berardi, A. Caivano, R. Ria et al., "Four proteins governing overangiogenic endothelial cell phenotype in patients with multiple myeloma are plausible therapeutic targets," Oncogene, vol. 31, no. 18, pp. 2258-2269, 2012.

[70] P. Leone, G. Di Lernia, A. G. Solimando et al., "Bone marrow endothelial cells sustain a tumor-specific CD8+ T cell subset with suppressive function in myeloma patients," Oncoimmunology, vol. 8, Article ID e1486949, 2019.

[71] M. Zarfati, I. Avivi, B. Brenner, T. Katz, and A. Aharon, "Extracellular vesicles of multiple myeloma cells utilize the proteasome inhibitor mechanism to moderate endothelial angiogenesis," Angiogenesis, vol. 22, no. 1, pp. 185-196, 2019.

[72] A. Vacca, C. Scavelli, V. Montefusco et al., "Thalidomide downregulates angiogenic genes in bone marrow endothelial cells of patients with active multiple myeloma," Journal of Clinical Oncology, vol. 23, no. 23, pp. 5334-5346, 2005.

[73] D. Ribatti, M. Moschetta, and A. Vacca, "Macrophages in multiple myeloma," Immunology Letters, vol. 161, no. 2, pp. 241-244, 2014.

[74] C. Scavelli, B. Nico, T. Cirulli et al., "Vasculogenic mimicry by bone marrow macrophages in patients with multiple myeloma," Oncogene, vol. 27, no. 5, pp. 663-674, 2008.

[75] O. Beyar-Katz, K. Magidey, A. Reiner-Benaim et al., "Proinflammatory macrophages promote multiple myeloma resistance to bortezomib therapy," Molecular Cancer Research, vol. 17, no. 11, pp. 2331-2340, 2019.

[76] H. Wang, W.-M. Hu, Z.-J. Xia et al., "High numbers of CD163+ tumor-associated macrophages correlate with poor prognosis in multiple myeloma patients receiving bortezomib-based regimens," Journal of Cancer, vol. 10, no. 14, pp. 3239-3245, 2019.
[77] J. Khalife, J. Ghose, M. Martella et al., "MiR-16 regulates crosstalk in NF- $\kappa \mathrm{B}$ tolerogenic inflammatory signaling between myeloma cells and bone marrow macrophages," JCI Insight, vol. 4, 2019.

[78] A. Rocci, C. C. Hofmeister, S. Geyer et al., "Circulating miRNA markers show promise as new prognosticators for multiple myeloma," Leukemia, vol. 28, no. 9, pp. 1922-1926, 2014.

[79] X. Lin, L. Yang, G. Wang et al., "Interleukin-32 $\alpha$ promotes the proliferation of multiple myeloma cells by inducing production of IL-6 in bone marrow stromal cells," Oncotarget, vol. 8, pp. 92841-92854, 2017.

[80] H. Yan, M. Dong, X. Liu et al., "Multiple myeloma cellderived IL-32 $\gamma$ increases the immunosuppressive function of macrophages by promoting indoleamine 2,3-dioxygenase (IDO) expression," Cancer Letters, vol. 446, pp. 38-48, 2019.

[81] D. Ribatti, A. Vacca, B. Nico et al., "Bone marrow angiogenesis and mast cell density increase simultaneously with progression of human multiple myeloma," British Journal of Cancer, vol. 79, no. 3-4, pp. 451-455, 1999.

[82] T. Nakayama, L. Yao, and G. Tosato, "Mast cell-derived angiopoietin-1 plays a critical role in the growth of plasma cell tumors," Journal of Clinical Investigation, vol. 114, no. 9, pp. 1317-1325, 2004.

[83] C. A. Pappa, G. Tsirakis, M. Devetzoglou et al., "Bone marrow mast cell density correlates with serum levels of VEGF and CXC chemokines ENA-78 and GRO- $\alpha$ in multiple myeloma," Tumor Biology, vol. 35, no. 6, pp. 5647-5651, 2014.

[84] C. A. Pappa, G. Tsirakis, E. Stavroulaki et al., "Mast cells influence the proliferation rate of myeloma plasma cells," Cancer Investigation, vol. 33, no. 4, pp. 137-141, 2015.

[85] M. Devetzoglou, R. Vyzoukaki, M. Kokonozaki et al., "High density of tryptase-positive mast cells in patients with multiple myeloma: correlation with parameters of disease activity," Tumor Biology, vol. 36, no. 11, pp. 8491-8497, 2015.

[86] D. Ribatti, R. Tamma, and A. Vacca, "Mast cells and angiogenesis in human plasma cell malignancies," International Journal of Molecular Sciences, vol. 20, no. 3, 2019.

[87] B. Nico, D. Mangieri, E. Crivellato, A. Vacca, and D. Ribatti, "Mast cells contribute to vasculogenic mimicry in multiple myeloma," Stem Cells and Development, vol. 17, no. 1, pp. 19-22, 2008.

[88] R. Vyzoukaki, G. Tsirakis, C. A. Pappa, M. Devetzoglou, M. Tzardi, and M. G. Alexandrakis, "The impact of mast cell density on the progression of bone disease in multiple myeloma patients," International Archives of Allergy and Immunology, vol. 168, no. 4, pp. 263-268, 2015.

[89] K. De Veirman, L. Rao, E. De Bruyne et al., "Cancer associated fibroblasts and tumor growth: focus on multiple myeloma," Cancers, vol. 6, no. 3, pp. 1363-1381, 2014.

[90] L. Raffaghello, A. Vacca, V. Pistoia, and D. Ribatti, "Cancer associated fibroblasts in hematological malignancies," Oncotarget, vol. 6, no. 5, pp. 2589-2603, 2015.

[91] T. Liu, C. Han, S. Wang et al., "Cancer-associated fibroblasts: an emerging target of anti-cancer immunotherapy," Journal of Hematology \& Oncology, vol. 12, no. 1, p. 86, 2019.

[92] L. Porcelli, R. M. Iacobazzi, R. Di Fonte et al., "CAFs and TGF- $\beta$ signaling activation by mast cells contribute to resistance to gemcitabine/nabpaclitaxel in pancreatic cancer," Cancers, vol. 11, no. 3, p. 330, 2019.

[93] A. Boccarelli, F. Esposito, M. Coluccia, M. A. Frassanito, A. Vacca, and N. Del Buono, "Improving knowledge on the activation of bone marrow fibroblasts in MGUS and MM 
disease through the automatic extraction of genes via a nonnegative matrix factorization approach on gene expression profiles," Journal of Translational Medicine, vol. 16, no. 1, p. 217, 2018.

[94] M. A. Frassanito, V. Desantis, L. Di Marzo et al., "Bone marrow fibroblasts overexpress miR-27b and miR-214 in step with multiple myeloma progression, dependent on tumour cell-derived exosomes," The Journal of Pathology, vol. 247, no. 2, pp. 241-253, 2019.

[95] V. Desantis, M. A. Frassanito, R. Tamma et al., "Rhu-Epo down-regulates pro-tumorigenic activity of cancer-associated fibroblasts in multiple myeloma," Annals of Hematology, vol. 97, no. 7, pp. 1251-1258, 2018.

[96] K. De Veirman, E. Van Valckenborgh, Q. Lahmar et al., "Myeloid-derived suppressor cells as therapeutic target in hematological malignancies," Frontiers in Oncology, vol. 4, p. 349, 2014.

[97] I. R. Ramachandran, A. Martner, A. Pisklakova et al., "Myeloid-derived suppressor cells regulate growth of multiple myeloma by inhibiting T cells in bone marrow," The Journal of Immunology, vol. 190, no. 7, pp. 3815-3823, 2013.

[98] K. De Veirman, J. A. Van Ginderachter, S. Lub et al., "Multiple myeloma induces Mcl-1 expression and survival of myeloid-derived suppressor cells," Oncotarget, vol. 6, pp. 10532-10547, 2015.

[99] A. M. Roccaro, A. Sacco, P. Maiso et al., "BM mesenchymal stromal cell-derived exosomes facilitate multiple myeloma progression," Journal of Clinical Investigation, vol. 123, no. 4, pp. 1542-1555, 2013.

[100] E. Malek, M. de Lima, J. J. Letterio et al., "Myeloid-derived suppressor cells: the green light for myeloma immune escape," Blood Reviews, vol. 30, no. 5, pp. 341-348, 2016.

[101] A. G. Solimando, A. Brandl, K. Mattenheimer et al., "JAM-A as a prognostic factor and new therapeutic target in multiple myeloma," Leukemia, vol. 32, no. 3, pp. 736-743, 2018.

[102] A. G. Solimando, D. Ribatti, A. Vacca, and H. Einsele, "Targeting B-cell non Hodgkin lymphoma: new and old tricks," Leukemia Research, vol. 42, pp. 93-104, 2016.

[103] H. Tamura, "Immunopathogenesis and immunotherapy of multiple myeloma," International Journal of Hematology, vol. 107, no. 3, pp. 278-285, 2018.

[104] L. Ai, S. Mu, C. Sun et al., "Myeloid-derived suppressor cells endow stem-like qualities to multiple myeloma cells by inducing piRNA-823 expression and DNMT3B activation," Molecular Cancer, vol. 18, p. 88, 2019.

[105] K. Nakamura, S. Kassem, A. Cleynen et al., "Dysregulated IL18 is a key driver of immunosuppression and a possible therapeutic target in the multiple myeloma microenvironment," Cancer Cell, vol. 33, no. 4, pp. 634-648, 2018.

[106] M. Ozerova and Y. Nefedova, "Estrogen promotes multiple myeloma through enhancing the immunosuppressive activity of MDSC," Leukemia \& Lymphoma, vol. 60, no. 6, pp. 1557-1562, 2019.

[107] C. Botta, A. Gullà, P. Correale, P. Tagliaferri, and P. Tassone, "Myeloid-derived suppressor cells in multiple myeloma: preclinical research and translational opportunities," Frontiers in Oncology, vol. 4, p. 348, 2014.

[108] P. Neri, P. Tagliaferri, M. T. Di Martino et al., "In vivo antimyeloma activity and modulation of gene expression profile induced by valproic acid, a histone deacetylase inhibitor," British Journal of Haematology, vol. 143, no. 143, pp. 520531, 2008.

[109] M. Cea, A. Cagnetta, M. Gobbi et al., "New insights into the treatment of multiple myeloma with histone deacetylase inhibitors," Current Pharmaceutical Design, vol. 19, no. 4, pp. 734-744, 2013.

[110] J.-I. Youn, V. Kumar, M. Collazo et al., "Epigenetic silencing of retinoblastoma gene regulates pathologic differentiation of myeloid cells in cancer," Nature Immunology, vol. 14, no. 3, pp. 211-220, 2013.

[111] O. Draghiciu, J. Lubbers, H. W. Nijman, and T. Daemen, "Myeloid derived suppressor cells-An overview of combat strategies to increase immunotherapy efficacy," Oncoimmunology, vol. 4, Article ID e954829, 2015.

[112] P. Sansone and J. Bromberg, "Targeting the interleukin-6/ $\mathrm{Jak} /$ stat pathway in human malignancies," Journal of Clinical Oncology, vol. 30, no. 9, pp. 1005-1014, 2012.

[113] Y. Nefedova, M. Fishman, S. Sherman, X. Wang, A. A. Beg, and D. I. Gabrilovich, "Mechanism of all-TransRetinoic acid effect on tumor-associated myeloid-derived suppressor cells," Cancer Research, vol. 67, no. 22, pp. 11021-11028, 2007.

[114] S. Kusmartsev, F. Cheng, B. Yu et al., "All-trans-retinoic acid eliminates immature myeloid cells from tumor-bearing mice and improves the effect of vaccination," Cancer Research, vol. 63, pp. 4441-4449, 2003.

[115] D. I. Gabrilovich and S. Nagaraj, "Myeloid-derived suppressor cells as regulators of the immune system," Nature Reviews Immunology, vol. 9, no. 3, pp. 162-174, 2009.

[116] N. Amodio, D. Bellizzi, M. Leotta et al., "miR-29b induces SOCS-1 expression by promoter demethylation and negatively regulates migration of multiple myeloma and endothelial cells," Cell Cycle, vol. 12, no. 23, pp. 3650-3662, 2013.

[117] J. Favaloro, T. Liyadipitiya, R. Brown et al., "Myeloid derived suppressor cells are numerically, functionally and phenotypically different in patients with multiple myeloma," Leukemia \& Lymphoma, vol. 55, no. 12, pp. 2893-2900, 2014.

[118] J. D. Veltman, M. E. H. Lambers, M. van Nimwegen et al., "COX-2 inhibition improves immunotherapy and is associated with decreased numbers of myeloid-derived suppressor cells in mesothelioma. Celecoxib influences MDSC function," BMC Cancer, vol. 10, p. 464, 2010.

[119] A. Argentiero, S. De Summa, R. Di Fonte et al., "Gene expression comparison between the lymph node-positive and -negative reveals a peculiar immune microenvironment signature and a theranostic role for WNT targeting in pancreatic ductal adenocarcinoma: a pilot study," Cancers, vol. 11, 2019.

[120] T. Reya and H. Clevers, "Wnt signalling in stem cells and cancer," Nature, vol. 434, no. 7035, pp. 843-850, 2005.

[121] P. Leone, S. Berardi, M. A. Frassanito et al., "Dendritic cells accumulate in the bone marrow of myeloma patients where they protect tumor plasma cells from CD8+ T-cell killing," Blood, vol. 126, no. 12, pp. 1443-1451, 2015.

[122] D. Alizadeh, E. Katsanis, and N. Larmonier, "Chemotherapeutic targeting of myeloid-derived suppressor cells," Oncoimmunology, vol. 3, Article ID e27359, 2014.

[123] F. Roth, A. C. De La Fuente, J. L. Vella, A. Zoso, L. Inverardi, and P. Serafini, "Aptamer-mediated blockade of IL4R triggers apoptosis of MDSCs and limits tumor progression," Cancer Research, vol. 72, no. 6, pp. 1373-1383, 2012.

[124] H. Qin, B. Lerman, I. Sakamaki et al., "Generation of a new therapeutic peptide that depletes myeloid-derived suppressor cells in tumor-bearing mice," Nature Medicine, vol. 20, no. 6, pp. 676-681, 2014.

[125] M.-V. Mateos, M.-T. Hernández, P. Giraldo et al., "Lenalidomide plus dexamethasone for high-risk smoldering 
multiple myeloma," New England Journal of Medicine, vol. 369, no. 5, pp. 438-447, 2013.

[126] K. Luptakova, J. Rosenblatt, B. Glotzbecker et al., "Lenalidomide enhances anti-myeloma cellular immunity," Cancer Immunology, Immunotherapy, vol. 62, no. 1, pp. 39-49, 2013.

[127] B. L. Ebert, N. Galili, P. Tamayo et al., "An erythroid differentiation signature predicts response to lenalidomide in myelodysplastic syndrome," PLoS Medicine, vol. 5, 2008.

[128] L. Lu, F. Payvandi, L. Wu et al., "The anti-cancer drug lenalidomide inhibits angiogenesis and metastasis via multiple inhibitory effects on endothelial cell function in normoxic and hypoxic conditions," Microvascular Research, vol. 77, no. 2, pp. 78-86, 2009.

[129] K. M. Kortüm, E. K. Mai, N. H. Hanafiah et al., "Targeted sequencing of refractory myeloma reveals a high incidence of mutations in CRBN and Ras pathway genes," Blood, vol. 128, no. 9, pp. 1226-1233, 2016.

[130] S. V. Rajkumar and R. A. Kyle, "Angiogenesis in multiple myeloma," Seminars in Oncology, vol. 28, no. 6, pp. 560-564, 2001.

[131] M. Moschetta, Y. Mishima, Y. Kawano et al., "Targeting vasculogenesis to prevent progression in multiple myeloma," Leukemia, vol. 30, no. 5, pp. 1103-1115, 2016.

[132] A. G. Solimando, M. C. Da Via', P. Leone et al., "Adhesionmediated multiple myeloma (MM) disease progression: junctional adhesion molecule a enhances angiogenesis and multiple myeloma dissemination and predicts poor survival," Blood, vol. 134, no. S1, p. 855, 2019.

[133] A. G. Solimando, M. C. Da Via', P. Borrelli et al., "Central function for JAM-a in multiple myeloma patients with extramedullary disease," Blood, vol. 132, no. S1, p. 4455, 2018.

[134] J. J. Biagi, L. Mileshkin, A. P. Grigg, D. W. Westerman, and H. M. Prince, "Efficacy of thalidomide therapy for extramedullary relapse of myeloma following allogeneic transplantation," Bone Marrow Transplantation, vol. 28, no. 12, pp. 1145-1150, 2001.

[135] M. A. Lawson, M. M. McDonald, N. Kovacic et al., "Osteoclasts control reactivation of dormant myeloma cells by remodelling the endosteal niche," Nature Communications, vol. 6 , no. 1, 2015.

[136] A. Palumbo, A. Chanan-Khan, K. Weisel et al., "Daratumumab, bortezomib, and dexamethasone for multiple myeloma," New England Journal of Medicine, vol. 375, no. 8, pp. 754-766, 2016.

[137] S. Lonial, M. Dimopoulos, A. Palumbo et al., "Elotuzumab therapy for relapsed or refractory multiple myeloma," New England Journal of Medicine, vol. 373, no. 7, pp. 621-631, 2015.

[138] J. Krejcik, T. Casneuf, I. S. Nijhof et al., "Daratumumab depletes CD38+ immune regulatory cells, promotes T-cell expansion, and skews $\mathrm{T}$-cell repertoire in multiple myeloma," Blood, vol. 128, no. 3, pp. 384-394, 2016.

[139] D. M. Benson, C. E. Bakan, A. Mishra et al., "The PD-1/PDL1 axis modulates the natural killer cell versus multiple myeloma effect: a therapeutic target for CT-011, a novel monoclonal anti-PD-1 antibody," Blood, vol. 116, no. 13, pp. 2286-2294, 2010.

[140] D. Atanackovic, S. V. Radhakrishnan, N. Bhardwaj, and T. Luetkens, "Chimeric Antigen Receptor (CAR) therapy for multiple myeloma," British Journal of Haematology, vol. 172, no. 5, pp. 685-698, 2016.

[141] F. Costa, R. Das, J. Kini Bailur, K. Dhodapkar, and M. V. Dhodapkar, "Checkpoint inhibition in myeloma: opportunities and challenges," Frontiers in Immunology, vol. 9, p. 2204, 2018.

[142] A. Badros, E. Hyjek, N. Ma et al., "Pembrolizumab, pomalidomide, and low-dose dexamethasone for relapsed/ refractory multiple myeloma," Blood, vol. 130, no. 10, pp. 1189-1197, 2017.

[143] T. Jelinek and R. Hajek, "PD-1/PD-L1 inhibitors in multiple myeloma: the present and the future," Oncoimmunology, vol. 5, no. 12, Article ID e1254856, 2016.

[144] A. Agazzi, "Report on the 56th ASH annual meeting, San Francisco, 4-9 December 2014," Ecancermedicalscience, vol. 9, p. 514, 2015.

[145] A. M. Roccaro, A. Sacco, W. G. Purschke et al., "SDF-1 inhibition targets the bone marrow niche for cancer therapy," Cell Reports, vol. 9, no. 1, pp. 118-128, 2014.

[146] M. C. Da Vià, A. G. Solimando, A. Garitano-Trojaola et al., "CIC mutation as a molecular mechanism of acquired resistance to combined BRAF-MEK inhibition in extramedullary multiple myeloma with central nervous system involvement," The Oncologist, vol. 25, no. 2, pp. 112-118, 2020.

[147] E. Dickreuter and N. Cordes, "The cancer cell adhesion resistome: mechanisms, targeting and translational approaches," Biological Chemistry, vol. 398, no. 7, pp. 721-735, 2017.

[148] Z.-W. Li and W. S. Dalton, "Tumor microenvironment and drug resistance in hematologic malignancies," Blood Reviews, vol. 20, no. 6, pp. 333-342, 2006.

[149] M. Rudelius, M. T. Rosenfeldt, E. Leich et al., "Inhibition of focal adhesion kinase overcomes resistance of mantle cell lymphoma to ibrutinib in the bone marrow microenvironment," Haematologica, vol. 103, no. 1, pp. 116-125, 2018.

[150] H. Ludwig, K. Weisel, M. T. Petrucci et al., "Olaptesed pegol, an anti-CXCL12/SDF-1 spiegelmer, alone and with bortezomib-dexamethasone in relapsed/refractory multiple myeloma: a phase IIa study," Leukemia, vol. 31, no. 4, pp. 997-1000, 2017.

[151] A. Argentiero, A. G. Solimando, O. Brunetti et al., "Skeletal metastases of unknown primary: biological landscape and clinical overview," Cancers, vol. 11, 2019.

[152] R. Vij, N. Horvath, A. Spencer et al., "An open-label, phase 2 trial of denosumab in the treatment of relapsed or plateauphase multiple myeloma," American Journal of Hematology, vol. 84, no. 10, pp. 650-656, 2009.

[153] G. J. Morgan, J. A. Child, W. M. Gregory et al., "Effects of zoledronic acid versus clodronic acid on skeletal morbidity in patients with newly diagnosed multiple myeloma (MRC myeloma IX): secondary outcomes from a randomised controlled trial," The Lancet Oncology, vol. 12, no. 8, pp. 743-752, 2011.

[154] G. D’Arena, P. G. Gobbi, C. Broglia et al., "Pamidronate versus observation in asymptomatic myeloma: final results with long-term follow-up of a randomized study," Leukemia \& Lymphoma, vol. 52, pp. 771-775, 2011.

[155] K. Gadó, G. Domján, H. Hegyesi, and A. Falus, "Role of interleukin-6 in the pathogenesis of multiple myeloma," Cell Biology International, vol. 24, no. 4, pp. 195-209, 2000.

[156] M. A. Frassanito, K. De Veirman, V. Desantis et al., "Halting pro-survival autophagy by TGF $\beta$ inhibition in bone marrow fibroblasts overcomes bortezomib resistance in multiple myeloma patients," Leukemia, vol. 30, no. 3, pp. 640-648, 2016.

[157] K. M. Abdulkadyrov, G. N. Salogub, N. K. Khuazheva et al., "Sotatercept in patients with osteolytic lesions of multiple 
myeloma," British Journal of Haematology, vol. 165, no. 6, pp. 814-823, 2014.

[158] W. Jelkmann, "Activin receptor ligand traps in chronic kidney disease," Current Opinion in Nephrology and Hypertension, vol. 27, no. 5, pp. 351-357, 2018.

[159] D. M. Gilkes, G. L. Semenza, and D. Wirtz, "Hypoxia and the extracellular matrix: drivers of tumour metastasis," Nature Reviews Cancer, vol. 14, no. 6, pp. 430-439, 2014.

[160] B. Muz, P. de la Puente, F. Azab, I. M. Ghobrial, and A. K. Azab, "Hypoxia promotes dissemination and colonization in new bone marrow niches in waldenström macroglobulinemia," Molecular Cancer Research, vol. 13, no. 2, pp. 263-272, 2015.

[161] P. Maiso, D. Huynh, M. Moschetta et al., "Metabolic signature identifies novel targets for drug resistance in multiple myeloma," Cancer Research, vol. 75, no. 10, pp. 2071-2082, 2015.

[162] J. Hu, D. R. Handisides, E. Van Valckenborgh et al., "Targeting the multiple myeloma hypoxic niche with TH-302, a hypoxia-activated prodrug," Blood, vol. 116, no. 9, pp. 1524-1527, 2010.

[163] J. P. Laubach, C.-J. Liu, N. S. Raje et al., "A phase I/II study of evofosfamide, a hypoxia-activated prodrug with or without bortezomib in subjects with relapsed/refractory multiple myeloma," Clinical Cancer Research, vol. 25, no. 2, pp. 478-486, 2019.

[164] J. Hu, E. Van Valckenborgh, D. Xu et al., "Synergistic induction of apoptosis in multiple myeloma cells by bortezomib and hypoxia-activated prodrug $\mathrm{TH}-302$, in vivo and in vitro," Molecular Cancer Therapeutics, vol. 12, no. 9, pp. 1763-1773, 2013. 